\title{
Der neue GOLD-Bericht 2011
}

\author{
The New GOLD Document
}

Autoren

Institute
P. Kardos ${ }^{1}$, C. Vogelmeier ${ }^{2}$, H. Worth ${ }^{3}$

${ }^{1}$ Gemeinschaftspraxis und Zentrum für Allergologie, Pneumologie, Schlafmedizin an der Klinik Maingau, Frankfurt/Main

${ }^{2}$ Universitätsklinikum Gießen und Marburg, Standort Marburg

${ }^{3}$ Medizinische Klinik 1, Klinikum Fürth eingereicht 21.6.2012 akzeptiert nach Revision 28. 6. 2012

\section{Bibliografie}

Dol http://dx.doi.org/ 10.1055/s-0032-1310053

Pneumologie 2012; 66: 493-496

(c) Georg Thieme Verlag KG Stuttgart · New York

ISSN 0934-8387

\section{Korrespondenzadresse Dr. med. Peter Kardos}

Gemeinschaftspraxis und Zentrum für Allergologie, Pneumologie, Schlafmedizin an der Klinik Maingau Scheffelstraße 33 60318 Frankfurt/Main Kardos@LungenpraxisMaingau.de
Am 30.12.2011 veröffentlichte GOLD (Global Initiative for Chronic Obstructive Lung Disease) die neue, wesentlich veränderte Version des Dokuments, das häufig Leitlinie genannt wird. Die Initiative selbst erhebt diesen Anspruch zwar nicht, die deutschen Leitlinien (NVL http://www.copd. versorgungsleitlinien.de/ und DGP [1]), aber auch weltweite nationale Leitlinien (zum Beispiel [2]: ATS/ERS „position paper“) basieren weitestgehend auf den GOLD-Empfehlungen.

Die äußerst erfolgreiche Initiative geht auf das Jahr 2001 zurück. Die vorletzte wesentliche Änderung von GOLD erfolgte 2006. Die GOLDEmpfehlungen zur Definition, Schweregrade und Stufentherapie werden weltweit nicht nur in der klinischen Praxis, sondern auch für das Design zahlreicher COPD-Studien - darunter auch aller Zulassungsstudien neuerer Medikamente - genützt.

In der vorliegenden Überarbeitung wird anstelle der auf Basis der FEV $_{1}$ durchgeführten Schweregradeinteilung eine Klassifikation mit den Komponenten Symptomatik, Lungenfunktion und Exazerbationshäufigkeit vorgeschlagen. Hiermit verbunden werden bisherige Empfehlungen zur Therapie modifiziert. Daher stellt sich für den deutschen Arzt die dringende Frage: Welchen Einfluss hat die Änderung auf meine diagnostische Einschätzung und mein Therapieverhalten?

\section{Problematik der bisherigen Schweregrade \\ $\nabla$}

Bislang erfolgte die Einteilung des Schweregrades fast ausschließlich anhand der $\mathrm{FEV}_{1}$, wenn man vom Sonderfall der sehr schweren COPD im GOLD-Stadium 4 mit $\mathrm{FEV}_{1}<50 \%$ des Sollwertes und der respiratorischen Insuffizienz absieht. Die Vorteile dieser Klassifikation lagen auf der Hand: Da die Diagnosestellung COPD ohnehin eine Spirometrie erfordert, lieferte diese diagnostische
Spirometrie automatisch den Schweregrad mit. Danach brauchte der Arzt (vielerorts, z. B. im Vereinigten Königreich, auch die COPD-Nurse) nur noch in der Tabelle „Therapy at each stage of COPD“ nachzuschauen, um die „richtigen“ Medikamente für den Patienten zu verschreiben. Obwohl die Einteilung in die einzelnen Schweregrade keinesfalls auf klassifizierbarer Evidenz beruhte, lieferte die Pharmaindustrie in Form von randomisierten kontrollierten Studien in den letzten 12 Jahren die Evidenz für die Wirksamkeit der wichtigsten Medikamente in diesem System, vor allem für mittelschwere, schwere oder sehr schwere COPD. Auf die eingeschränkte externe Validität dieser Studien für die tägliche Praxis muss allerdings hingewiesen werden $[3,4]$. Ein weiteres Problem ist, dass diese Schweregradeinteilung den Zustand eines COPD-Patienten nur inkomplett abbildet. Seit 2004 entstanden komplexere Schweregradeinteilungen [5,6], der BODEIndex (Body mass index, Obstruction, Dyspnoea, Exercise $=6$ Min. Gehtest) [7] ist hierfür das bekannteste Beispiel. Er korreliert besser mit der Mortalität als die darin enthaltene $\mathrm{FEV}_{1}$, als einzelne Kenngröße betrachtet. Auch die Korrelation zwischen $\mathrm{FEV}_{1}$ und Lebensqualität, gemessen mit dem St. George's Respiratory Questionnaire (SGRQ), ist zwar signifikant, aber nicht sehr eng. Mit anderen Worten: Weder SGRQ noch FEV charakterisieren den COPD-Patienten vollständig. Bei einer chronischen Erkrankung wie COPD, deren Behandlung zwar eine erhebliche Symptomlinderung, aber keine Heilung bringen kann, rücken zudem patientennahe Messparameter, „PRO's, patient reported outcomes“, in den Vordergrund. Nicht nur für klinische Zulassungsstudien, sondern auch für täglich erforderliche klinische Entscheidungen [8]. Die Subjektivität ist ihr Vorteil, aber zugleich auch ihr Nachteil. 


\begin{tabular}{|llllll|}
\hline Typ & $\begin{array}{l}\text { Charakteristika: } \\
\text { Symptome } \\
\text { Exazerbationen }\end{array}$ & $\begin{array}{l}\text { Spirometrische } \\
\text { Klassifikation } \\
\text { der Obstruktion }\end{array}$ & $\begin{array}{l}\text { Exazerbationen } \\
\text { pro Jahr }\end{array}$ & $\begin{array}{l}\text { Symptome } \\
\text { CAT }\end{array}$ & mMRC \\
\hline A & $\begin{array}{l}\text { niedriges Risiko } \\
\text { wenig Symptome }\end{array}$ & GOLD 1/GOLD 2 & $\leq 1$ & $\geq 10$ & $2+$ \\
\hline B & $\begin{array}{l}\text { niedriges Risiko } \\
\text { mehr Symptome }\end{array}$ & GOLD 1/GOLD 2 & $\leq 1$ & $<1$ & $\leq 1$ \\
\hline C & $\begin{array}{l}\text { hohes Risiko } \\
\text { wenig Symptome } \\
\text { hohes Risiko } \\
\text { mehr Symptome }\end{array}$ & GOLD 3/GOLD 4 & $2+$ & $\geq 10$ & $2+$ \\
\hline D & GOLD 3/GOLD 4 & $2+$ & & $<10$ \\
\hline
\end{tabular}

Tab. 1 Kombinierte klinische Schweregradeinteilung der COPD.

\section{Die neue GOLD-2011-Lösung für die Schweregrade \\ $\nabla$}

Um der Komplexität der Schwere der Erkrankung besser Rechnung zu tragen, hat GOLD 2011 eine Kombination von 4 Kenngrößen eingeführt:

- Symptome

- Funktionelle Beeinträchtigung (Spirometrie)

- Exazerbationsrisiko

- Komorbiditäten

Es erwies sich als schwierig, die klinische Vielfalt in kurze, übersichtliche Schemata zu drängen. Denn selbst wenn die klinische Wirklichkeit in binäre Antworten von kategorischen Variablen (schwer oder leicht, ja oder nein) subsummiert wird, obwohl es sich um kontinuierliche Variablen handelt, gäbe es in diesem System 16 Schweregrade (A-P)!

Aspekte der Praktikabilität führten daher zu der vorliegenden vierstufigen klinischen Schweregradeinteilung der COPD (A-D), die auf der Kombination der Ausprägung der Symptome und des Risikos zukünftiger (zu vermeidender) Exazerbationen basiert. Sie sollen auch die erforderliche Therapie bestimmen.

1. Zwei validierte Fragebögen (mMRC, modified Medical-Research-Council-Dyspnoe-Fragebogen mit 0-4 Punkten, oder CAT, COPD assessment test mit 8 Fragen, je 0-5 Punkte) sollen zur „objektiven“ Beurteilung des subjektiven Befindens des Patienten dienen. 0-1 Punkte in mMRC und 0-9 Punkte in CAT stehen für wenig ausgeprägte Symptome, höhere Scores für stärker ausgeprägte Symptome.

2. Wer mehr als eine Exazerbation im vergangenen Jahr gehabt hat, weist ein hohes Risiko für Exazerbation auf. Das Exazerbationsrisiko steigt aber, wie lange bekannt, auch mit zunehmender Flusslimitierung. In der neuen GOLD-Empfehlung dient die spirometrische Klassifizierung der Schwere der Obstruktion (die bisherigen Kategorien leicht, mittelschwer, schwer und sehr schwer bleiben unverändert) nicht mehr zur Einschätzung des Schwergerades der Erkrankung, sondern im vierstufigen System A-D ausschließlich zur Einschätzung des Exazerbationsrisikos. Im Gegensatz zu der Symptommessung, wo ausdrücklich nur eines der empfohlenen Messinstrumente (CAT oder mMRC) eingesetzt werden sollte, soll das Exazerbationsrisiko sowohl anhand der spirometrischen als auch der anamnestischen Methode beurteilt werden. Der klinische Schweregrad der COPD wird dann anhand des schlechteren Wertes beurteilt. Durch diesen „Kunstgriff“ gelang es, die alte spirometrische Schweregradeinteilung in die neuen Empfehlungen zu integrieren: Patienten mit schwerer und sehr schwerer Obstruktion gelangen - unabhängig von dem im individuellen Fall gelegentlich abweichendem Exazerbationsrisiko - in die Kategorien GOLD C und D.

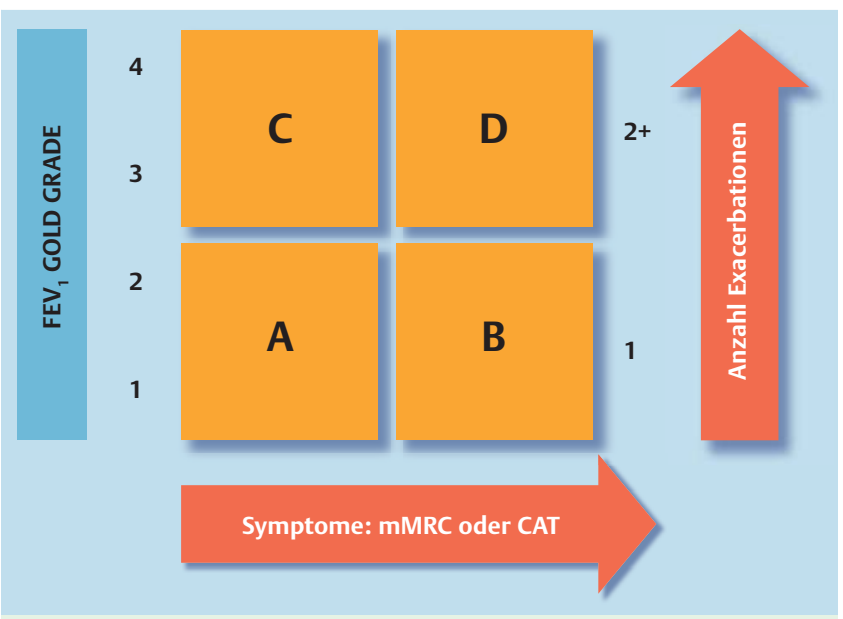

Abb. 1 Die neuen GOLD-2011-Schweregrade.

Der klinische Schweregrad wird wie folgt beurteilt: A (wenig Symptome, geringes Risiko), B (ausgeprägte Symptomatik, geringes Risiko), C (geringe Symptomatik, hohes Risiko) und D (ausgeprägte Symptomatik, hohes Risiko). ( $\bullet$ Tab. 1 und $\bullet$ Abb.1)

\section{Die neuen GOLD-2011-Empfehlungen für die Therapie $\nabla$}

Es wurden Medikamente der ersten und zweiten Wahl sowie alternative Medikamente definiert ( $\bullet$ Tab.2). Die Übersetzung „first choice“ als erste Wahl ist irreführend. Es handelt sich hier nicht um die besten („first line“) Medikamente, sondern um solche, die bei der ersten Konsultation eingesetzt werden können. Wenn sie nicht ausreichen, greift man in einer zweiten Auswahl (zweite Konsultation) auf die wortwörtlich zu nehmenden „Zweite-Wahl“-Medikamente. Die alternativen Therapien werden als die verfügbare Alternative verstanden, wenn erst- oder zweitrangige Medikamente nicht vorhanden oder nicht erreichbar („unavailable or unaffordable“) sind. Sie können laut GOLD auch mit den Medikamenten der ersten oder zweiten Wahl kombiniert werden. Diese Alternativen tragen der Situation in Entwicklungsländern Rechnung. 


\begin{tabular}{|c|c|c|c|}
\hline Typ & Erste Wahl & Zweite Wahl & Alternative \\
\hline A & $\begin{array}{l}\text { SABA oder SAMA } \\
\text { bei Bedarf }\end{array}$ & $\begin{array}{l}\text { SABA + SAMA } \\
\text { LABA oder LAMA }\end{array}$ & Theophyllin \\
\hline B & LABA oder LAMA & $L A B A+L A M A$ & Theophyllin, SABA und/oder SAMA \\
\hline C & LABA/ICS oder LAMA & $\begin{array}{l}\text { LABA + LAMA } \\
\text { ICS + LAMA }\end{array}$ & $\begin{array}{l}\text { Theophyllin } \\
\text { SABA und/oder SAMA } \\
\text { Roflumilast }^{1}\end{array}$ \\
\hline D & LABA/ICS oder LAMA & $\begin{array}{l}\text { LABA/ICS + LAMA } \\
\text { LABA/ICS + Roflumilast }^{1} \\
\text { LAMA + Roflumilast }^{1}\end{array}$ & $\begin{array}{l}\text { Theophyllin } \\
\text { SABA und/oder SAMA } \\
\text { Carbocystein }\end{array}$ \\
\hline
\end{tabular}

Tab. 2 Das neue GOLD-Stufenschema für die initiale Therapie.

SABA: kurz wirksamer Beta 2 -Agonist; SAMA: kurz wirksames Anticholinergikum; LAMA: lang wirksames Anticholinergikum;

LABA: lang wirksamer Beta 2 -Agonist; ICS: inhalatives Kortikosteroid

${ }^{1}$ Nur bei Bronchitis-Phänotyp (Husten und Auswurf)

\section{Kommentar zum Stufenschema}

$\nabla$

1. Es muss festgestellt werden, dass für die Einteilung in die alten, uns lieb gewordenen rein spirometrischen COPD-Schweregrade 1 - 4 ebenso wenig Evidenz gibt, wie für das neue Konstrukt A-D, dass auf der Meinung ausgewiesener internationaler Experten beruht. Nach dem von der ATS adaptierten GRADE-System [9] bedeutet dies: Keine Evidenz.

2. Die zur Schweregradeinteilung nun obligate Beurteilung der Symptome anhand von Fragebögen (CAT oder mMRC) entspricht nicht der täglichen Praxis in Deutschland und könnte ein Hindernis zur Implementierung einer solchen Empfehlung darstellen. Nun hat das GOLD-Komitee anlässlich der internationalen Konferenz in San Francisco (Mai 2012) diese Empfehlung in einem Vortrag „relativiert“: Die Fragebögen seien nur ein fakultatives Hilfsmittel zur Erhebung der Symptome.

3. Die Empfehlungen zur medikamentösen Therapie stützen sich - soweit vorhanden - auf Evidenz aus klinischen Studien. Es gibt starke Evidenz aus zahlreichen großen Studien, dass lang wirksame Bronchodilatatoren, inhalative Kortikosteroide und PDE4-Hemmer und, weniger gut belegt, auch als Mukopharmaka verwendete Antioxidanzien das Exazerbationsrisiko senken und vor allem die lang wirksamen Bronchodilatatoren die Symptome lindern. Es ist nicht die Aufgabe dieser Stellungnahme, die vorhandene Evidenz zu sichten. Über die additiven Effekte kombinierter Pharmakotherapien für die einzelnen neuen Schweregrade A-D ist die Evidenzlage bereits deutlich schwächer. Der Preis für die „heimliche“ Einbindung der spirometrischen Schweregrade der Obstruktion ist eine Ausdünnung der Evidenzbasis für die neue abgestufte Therapieempfehlung, da sie auch für in Wirklichkeit nicht exazerbierenden Patienten diejenige Therapie empfiehlt, die nach Studienlage auf die Prävention der Exazerbationen ausgerichtet ist (ICS, Roflumilast, teilweise auch Tiotropium, um über die „alternative Empfehlung“ Carbocystein ganz zu schweigen). Die Kombination „alternativer“ Medikamente wie Theophyllin mit einigen Medikamenten der zweiten Wahl (Roflumilast) ist ebenfalls ohne Evidenz, in diesem Extremfall wahrscheinlich auch ohne wissenschaftliche Rationale.

\section{Was hat sich mit GOLD 2011 geändert für die praktische Therapie?}

Die bisherige starre Therapieempfehlung ist deutlich aufgelockert worden; dies ist aber für den deutschen Leser auf den ersten Blick möglicherweise nicht offensichtlich. Betreffend die „Erste Wahl“, handelt es sich um eine initiale Therapie, die nach dem Verbrauch der ersten N1-Packung angepasst werden sollte. Nur so ist es verständlich, dass zum Beispiel eine Monotherapie mit Tiotropium sowohl für den leichtesten (A als 2.Wahl) als auch für den höchsten Schweregrad (D als 1.Wahl) empfohlen wird. Nach dieser initialen Therapie, die eine Überbehandlung auf jeder Stufe vermeidet, ist dann praktisch bei der zweiten Auswahl der Medikamente jede erdenkliche Kombination möglich. Die Medikamente bleiben ohnehin dieselben. Das neue Schema erhöht somit unsere Therapiefreiheit in der Behandlung.

So ist für die schweren Fälle $\mathrm{C}+\mathrm{D}$ (Patienten mit stark eingeschränkter Lungenfunktion und/oder häufigen Exazerbationen) praktisch jede Kombination möglich (und kann auch im individuellen Fall sinnvoll sein). Es verstößt auch nicht mehr gegen die Leitlinie, für den jüngeren Patienten mit wenig Symptomen (Schweregrad A) einen lang wirksamen Bronchodilatator zu verordnen, wenn er zum Beispiel Ausdauersport treibt.

Die erweiterte Therapiefreiheit ist für den Pneumologen ein Gewinn, kann aber für den Allgemeinarzt, der die Mehrzahl der COPD-Patienten behandelt, eher ein Problem bei der Auswahl der Medikamente darstellen. Die klaren Empfehlungen für eine Therapieeskalation nach dem alten Stufenschema wurden der Therapiefreiheit geopfert. Eine Therapieeskalation (horizontal von der 1.Wahl zur 2. Wahl; oder vertikal von Stufe A zu Stufe B, C oder D) macht keinen Sinn mehr.

Ein weiterer wichtiger Punkt ist, dass die Verordnungsfähigkeit der Medikamente zu Lasten der gesetzlichen Krankenkassen und eigentlich auch der privaten Krankenversicherungen (zugelassene Indikation, „label“) selbst dann nicht geändert wäre, wenn die neuen GOLD-Empfehlungen in die nationale(n) Leitlinie(n) übernommen werden würden. So bleibt zum Beispiel eine ICS/LABAKombination für GOLD-C- und D-Patienten, die nicht anhand der Zahl der Exazerbationen, sondern alleine durch den hohen Schweregrad in diese Kategorie gelangten, „off label“. Nicht einmal alle verfügbaren ICS/LABA-Kombinationen und -Dosierungen sind für COPD zugelassen; zu den Kuriositäten gehört, dass Fluticason/Salmeterol als Discus zugelassen, jedoch als Dosieraerosol in der gleichen Dosierung „off label“ ist. 


\section{Fazit}

Es ist ein großer Verdienst des neuen GOLD-Reports, dass entgegen dem bisherigen Trend der Umfang gegenüber 2009 um $20 \%$ abgenommen hat (74 Druckseiten gegenüber 90 Druckseiten).

Die neue Schweregradeinteilung A-D muss sich in der Praxis noch bewähren. Sie hat aber zwei unübersehbare Vorteile:

1. Die Sicht des stark beschäftigten Arztes, der sich häufig auf die zwei wichtigsten Abbildungen einer Leitlinie beschränkt (Schweregrade und medikamentöse Therapieempfehlung) - wird mit dem neuen Schema ( $\bullet$ Abb. 1) auf die Beschwerden des Patienten fokussiert.

2. GOLD 2011 gibt das starre, der Individualität des Patienten nicht immer gerecht werdende $\mathrm{FEV}_{1}$-abhängige „Therapiediktat" zugunsten einer flexiblen, mehr individualisierbaren Therapieempfehlung auf; selbst dann, wenn diese Liberalisierung eindeutig zu Lasten der statistischen Evidenz und der klaren Anweisung zur ggf. notwendigen Empfehlung zur Therapieeskalation geht.

\section{Interessenkonflikt}

Die Autoren geben an, dass sie Beratungs- und Vortragshonorare sowie Reisekostenerstattung von verschiedenen Firmen erhalten haben, die Produkte vertreiben, die im GOLD-Dokument erwähnt werden. Es wird in dieser Stellungnahme nur die aktuelle GOLD-Empfehlung aufgeführt, keine eigene Empfehlung ausgesprochen. Insofern bestehen keine Interessenkonflikte.

\section{Literatur}

1 Vogelmeier C, Buhl R, Criée CP et al. Leitlinie der Deutschen Atemwegsliga und der Deutschen Gesellschaft für Pneumologie und Beatmungsmedizin zur Diagnostik und Therapie von Patienten mit chronisch obstruktiver Bronchitis und Lungenemphysem (COPD). Pneumologie 2007; 61: e1-40

2 Celli BR, MacNee W, Agusti A et al. Standards for the diagnosis and treatment of patients with COPD: a summary of the ATS/ERS position paper. Eur Respir J 2004; 23: 932 - 946

3 Witt C, Tresz A, Wegscheider K. Externer Validität auf der Spur. Dtsch Arztebl 2011; 108: 2468-2474

4 Travers J, Marsh S, Caldwell B et al. External validity of randomized controlled trials in COPD. Respir Med 2007; 101: 1313-1320

5 Puhan MA, Garcia-Aymerich J, Frey $M$ et al. Expansion of the prognostic assessment of patients with chronic obstructive pulmonary disease: the updated BODE index and the ADO index. Lancet 2009; 374: 704711

6 Jones RC, Donaldson GC, Chavannes NH et al. Derivation and validation of a composite index of severity in chronic obstructive pulmonary disease: the DOSE Index. Am J Respir Crit Care Med 2009; 180: 11891195

7 Celli BR, Cote CG, Marin JM et al. The Body-Mass Index, Airflow Obstruction, Dyspnea, and Exercise Capacity Index in Chronic Obstructive Pulmonary Disease. N Engl J Med 2004; 350: 1005-1012

8 Snyder CF, Aaronson NK. Use of patient-reported outcomes in clinical practice. Lancet 2009; 374: 369-370

9 Schunemann HJ, Jaeschke R, Cook DJ et al. An official ATS statement: grading the quality of evidence and strength of recommendations in ATS guidelines and recommendations. Am J Respir Crit Care Med 2006; 174: $605-614$ 